\section{Odour and Chemical Constitution}

Nothrng is known of the mechanism by which small amounts of odorous material can trigger nervous impulses leading to smell sensations, because the characteristic odour of a substance has not hitherto been securely correlated with any other characteristic property of its molecules.

Some years ago, G. M. Dyson ${ }^{1}$ concluded that odour must be related to a characteristic molecular vibration pattern, and he assigned certain odours to certain Raman frequencies ("osmic frequencies") in the general range $1,500-3,000 \mathrm{~cm}^{-1}$. In this region, Raman lines are correlated strongly with particular functional groups $(\mathrm{OH}, \mathrm{SH}, \mathrm{CO}$, etc.), so that if odour could be correlated with these frequencies it could equally well be correlated with the corresponding functional groups and there would be no need for a vibrational theory of odour. On the other hand, Raman lines below about $1,000 \mathrm{~cm}^{-1}$ appear to depend upon the whole configuration of the molecule more than on the constituent groups. Odour also appears to depend more on the whole configuration of the molecule, so that a correlation of odour with vibration would be more reasonable at low frequencies than at high. Moreover, the Planck formula shows that only normal vibrations with a wave number considerably less than $1,000 \mathrm{~cm} .^{-1}$ have a significant probability of being active at body temperature.

Dyson's "osmic frequencies" are therefore excluded on both chemical and quantum grounds, and any correlation between odours and molecular vibration patterns must be looked for at frequencies below about $700 \mathrm{~cm} .^{-1}$ corresponding to absorption in the infra-red beyond about 15 microns. This region does not seem to have been previously searched for odour correlations.

A panel of fifteen observers was used to select sixteen compounds with an odour resembling that of nitrobenzene. Of these compounds, all but one (nitrothiophene) were benzene derivatives, and all but one (isocoumarane) had multiple bonds in the sidechain conjugated with the ring. Apart from this general similarity of type, the compounds had such varied functional groups as nitro, nitrile, aldehyde, ketone, ester, etc. When the low-frequency Raman shifts of these compounds were scrutinized, there was some evidence of a statistically significant correlation between the odour and the pattern of the Raman lines. This work is being reported in detail elsewhere.

The Raman frequencies below $800 \mathrm{~cm}^{-1}$ of three of these compounds (nitrobenzene, nitrothiophene and benzonitrile) are shown in Fig. 1. A search of the literature for substances with unrelated structures but similar Raman spectra showed that butyronitrile met the requirements fairly well. In the opinion of six observers, its odour is somewhat almond-like. The successful selection at the first attempt of an aliphatic compound with the same general type of odour as a group of sixteen aromatic substances is

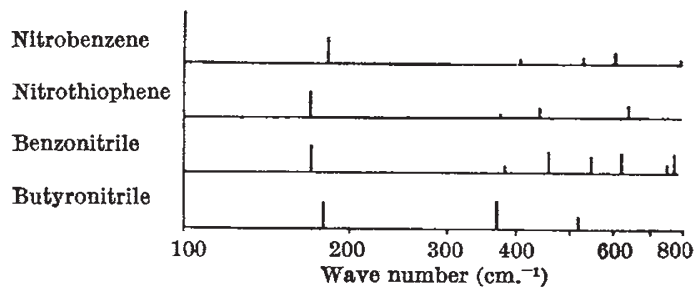

Fig. 1 something which no other theory of the physical basis of odour would enable one to do.

A correlation of odour with the pattern of molecular vibrations may therefore be possible if the frequencyrange below $1,000 \mathrm{~cm}^{-1}$ is studied. The Raman effect is at the present the most convenient tool for the purpose.

Division of Chemistry,

R. H. WRIGHT

British Columbia Research Council, Vancouver 8, B.C.

Jan. 27.

1 Dyson, G. M., Perf. Essent. Oil Record, 19, 456 (1928); 28, 13 (1937); Chem. and Indust., 16, 647 (1938).

IN the early 'twenties when the first work on this aspect of osmics was under active consideration our knowledge of the Raman lines below $1,000 \mathrm{~cm} .^{-1}$ was rudimentary, and while correlation between structural groups and the higher frequencies is now fairly well established, the need for a vibrational theory of odour does not, in my view, automatically disappear.

The interesting results established by $\mathrm{Mr}$. $\mathrm{R} . \mathrm{H}$. Wright prove, if they prove anything, that the original hypothesis correlating odour specificity with molecular vibration was sound in principle, but that I was wrong in assuming the frequencies concerned to be those in the higher Raman ranges. He has made a valuable advance in this field by focusing attention on the significance of the lower frequencies ; they relate to the molecule as a whole and this, in itself, is an attractive development of the original thesis. There are, however, several groups of compounds which must come under review before the theory can be fully accepted: I refer in particular (a) to the group $R . \mathrm{C}_{6} \mathrm{H}_{4} . \mathrm{NCS}$ which, when $R=\mathrm{H}$, $o-\mathrm{CH}_{3}$ or $o-\mathrm{Cl}$, retains the mustard-like odour associated with allylthiocarbimide, but when $R=\mathrm{CH}_{3}$, $p-\mathrm{Cl}, p-\mathrm{OCH}_{3}$, acquires a strong odour resembling anisaldehyde (en passant, 4-cyanophenylthiocarbimide has a strong almond component to its odour); (b) the 'camphor-like' groups in which such diverse substances as camphor, hexachloroethene, benzene hexachloride and perchloronaphthalene have closely related odours; and (c) the 'tea-rose' thiazoles of Bogert, which have odours reminiscent of phenyl acetaldehyde or the esters of phenylacetic acid.

1 Burton Walks,

G. Matcolm Drson

Loughborough.

\section{Effect of Flight on Behaviour of Aphis fabae Scop.}

After a preliminary period of quiescence newly moulted alate alienicolae of Aphis fabae Scop. take flight, in suitable physical conditions, whatever the quality of the host they are on. When they alight on a host plant after a sufficient flight they do not take off again immediately ${ }^{1}$ as they do before sufficient flight ${ }^{2}$, and they may settle down and bear young. Moericke ${ }^{2}$ thought that this indicated two phases in the life of alate aphids, the Zugphase and Befallsphase, and Müller and Unger ${ }^{3}$ attributed the transition from one to the other to exhaustion. The following experiments show that the change can be brought about by flight itself, and that it is not necessarily permanent.

The experiments were carried out in a greenhouse and the laboratory at temperatures between 20 and 